\title{
Case-matched comparative analysis of spinal deformity correction in arthrogryposis multiplex congenita versus adolescent idiopathic scoliosis
}

\author{
*Liang Xu, MD, Zhonghui Chen, MD, Yong Qiu, MD, Xi Chen, MD, Song Li, MD, Changzhi Du, MD, \\ Qingshuang Zhou, MD, and Xu Sun, MD
}

Department of Spine Surgery, Drum Tower Hospital, Nanjing University Medical School, Nanjing, China

OBJECTIVE As scoliosis in arthrogryposis multiplex congenita (AMC) is unusual and the number of cases reviewed in previous studies is also relatively small, no previous study exists that has directly compared the results of spinal deformity correction between AMC and adolescent idiopathic scoliosis (AIS) patients. The aim of this study was to compare the radiographic and clinical outcomes of surgical correction of spinal deformity associated with AMC versus AIS.

METHODS Twenty-four adolescents with AMC were matched with 48 AIS patients in terms of Cobb angle of main curve, curve pattern, sex, age at surgery, Risser grade, and length of follow-up. Patients in both groups underwent posterior-only spinal correction and fusion procedures. The surgical outcomes and complications were analyzed and compared between the 2 groups.

RESULTS In comparison to the AIS group, the AMC group had a significantly longer mean operation time (5.6 vs 4.4 hours, $p=0.002$ ), more blood loss ( $1620 \pm 250 \mathrm{ml} v 840 \pm 260 \mathrm{ml}, p<0.001)$, and more fusion levels (14.1 \pm 2.3 levels vs $12.4 \pm 2.5$ levels, $p=0.007)$ as well as a lower correction rate $(44.3 \% \pm 11.1 \%$ vs $70.8 \% \pm 12.4 \%, p<0.001)$ and a higher rate of loss of correction $(5.0 \% \pm 3.1 \%$ vs $2.1 \% \pm 1.9 \%, p<0.001)$. Nine patients in the AMC group had preoperative pelvic obliquity, which was corrected from a mean of $14.2^{\circ} \pm 8.4^{\circ}$ to a mean of $4.3^{\circ} \pm 3.2^{\circ}(p<0.001)$ after the surgery. The thoracic lordosis and sagittal vertical axis were significantly improved in the AMC group. Notably, however, the AMC group was found to have higher rates of screw malpositioning ( $15.9 \%$ vs $9.5 \%, p=0.002)$ and complications ( $8 / 24$ [33.3\%] vs $4 / 48[8.3 \%], p=0.016$ ) as compared to the AIS group.

CONCLUSIONS Correction of AMC-associated scoliosis tends to require a longer operating time and involve more fusion levels but results in less correction, more blood loss, and more complications, in comparison with AIS. In addition, more attention should be paid to pelvic obliquity and sagittal hyperlordosis in AMC patients.

https://thejns.org/doi/abs/10.3171/2018.7.PEDS18347

KEYWORDS arthrogryposis multiplex congenita; adolescent idiopathic scoliosis; posterior instrumentation; correction outcome; spine

$\mathrm{A}$ RTHROGRYPOSIS multiplex congenita (AMC) is a nonprogressive disorder, present at birth and characterized by multiple joint contractures and defective muscles, with normal sensation. ${ }^{4,7}$ In general, AMC mainly involves the limbs, but sometimes the spine is also involved..$^{12}$ The reported incidence of scoliosis varies from $30 \%$ to $67 \% .^{13,16,22}$ In most cases, the curves tend to progress rapidly and become stiff at an early age..$^{13}$ Although various curve patterns have been reported, a single, C- shaped, thoracolumbar curve, often associated with pelvic obliquity, is the most common curve type ${ }^{6,15}$ As brace treatment is rarely successful, progressive curves require surgical intervention. ${ }^{5}$ For young children, a growing rod technique is recommended, ${ }^{2,10}$ whereas spinal fusion surgery is typically performed in teenagers..$^{19,22}$

So far, only a few articles have described the surgical treatment of scoliosis in AMC, and the number of cases reviewed is also relatively small. ${ }^{11}$ Currently there are

ABBREVIATIONS AIS = adolescent idiopathic scoliosis; $\mathrm{AMC}=$ arthrogryposis multiplex congenita; $\mathrm{C7PL}=\mathrm{C7}$ plumb line; $\mathrm{CSVL}=\mathrm{center}$ sacral vertical line; $\mathrm{SRS}=\mathrm{Sco}-$ liosis Research Society; SVA = sagittal vertical axis.

SUBMITTED June 3, 2018. ACCEPTED July 18, 2018.

INCLUDE WHEN CITING Published online October 12, 2018; DOI: 10.3171/2018.7.PEDS18347.

${ }^{*}$ L.X. and Z.C. contributed equally to this work. 
no established practice guidelines for the surgical treatment of AMC-associated scoliosis. Therefore, the surgical regimens for spine deformities in patients with AMC are usually derived from the experience in patients with adolescent idiopathic scoliosis (AIS). However, these 2 pathologies differ dramatically in terms of both management and prognosis. To our knowledge, there has been no study that directly compared patients with AMC and patients with AIS who have undergone spinal fusion. The current study retrospectively reviewed the surgical outcomes and complications of treatment in patients with AMC-associated scoliosis and a well-matched cohort of patients with AIS, aiming to illustrate the differences and to identify areas for potential improvement.

\section{Methods \\ Patients}

After approval was obtained from our institutional review board, we retrospectively identified $41 \mathrm{AMC}$ patients from our scoliosis database who had undergone surgical treatment for scoliosis between July 2006 and July 2017. Inclusion criteria for the AMC group were as follows: 1) age between 10 and 18 years, 2) Cobb angle of the major curve ranging from $50^{\circ}$ to $100^{\circ}, 3$ ) single-stage posterioronly correction and fusion surgery, and 4) at least 2 years of postoperative follow-up. Patients who were nonambulatory preoperatively or had congenital kyphosis or incomplete radiographic data were excluded. A database of patients with AIS who had undergone spinal fusion was also reviewed. A group of AIS patients was selected who were well matched to the AMC group at a 2:1 ratio in terms of curve pattern, sex, Cobb angle of main curve (within $5^{\circ}$ ), age at surgery (within 1 year), Risser grade (within 1), and length of follow-up (within 6 months). Factors other than these were not considered in the matching process.

\section{Surgical Intervention}

Patients in both groups underwent correction surgery with posterior-only instrumentation and careful neuromonitoring. All AMC patients and those AIS patients with curve flexibility less than $30 \%$ underwent Ponte osteotomies, which consisted of resection of the posterior elements through the facet joints and posterior ligaments at the apex and at the superior and inferior adjacent levels. ${ }^{9,20}$ After osteotomy, rods were contoured to normal sagittal alignment and then were attached to the screws at the proximal and distal ends. Once the rod was anchored at the bottom screw with a normal sagittal profile, it was introduced to the remaining screws sequentially from the ends to the apex of the curve. Gradual correction was performed with a combination of translation and de-rotation techniques followed by convex compression and concave distraction maneuvers.

\section{Radiographic and Clinical Evaluations}

All patients underwent radiographic and clinical evaluation preoperatively and immediately postoperatively; clinical and radiographic follow-up was routinely scheduled for 3, 6, and 12 months after surgery and then annually thereafter. All patients in this study had at least 2 years of postoperative follow-up, per the inclusion criteria, and findings at the most recent follow-up examination were used for purposes of analysis. The coronal deformity angular ratio was measured as the curve magnitude divided by number of levels of the deformity. The distance between the C7 plumb line (C7PL) and the center sacral vertical line (CSVL) was measured to assess coronal balance, and the sagittal vertical axis (SVA) was measured to assess sagittal balance. Side-bending radiographs of the whole spine were used to evaluate spinal flexibility in the coronal plane.

Postoperative CT scans were routinely performed in all cases to detect any malpositioned pedicle screws potentially causing neural or vascular injury or impingement. Using the established grading system, ${ }^{1,17,23}$ pedicle perforations were classified as medial, lateral, or anterior and categorized into one of 4 grades: grade $1, \leq 2 \mathrm{~mm}$; grade 2, 2.1-4.0 mm; grade 3, 4.1-6.0 mm; grade 4, $\geq 6.1 \mathrm{~mm}$. Pedicle screw position was also classified as acceptable (fully contained screws or screws with $\leq 2 \mathrm{~mm}$ of medial wall perforation or $\leq 6 \mathrm{~mm}$ of lateral wall perforation and without impingement of visceral organs) or unacceptable.

Scores on the Scoliosis Research Society (SRS)-22 patients' questionnaire, which contains 5 domains (including pain, self-image, function, satisfaction, and mental health), were used to evaluate the functional outcomes. Complications were also recorded.

\section{Statistical Analyses}

All data were analyzed with IBM SPSS software (version 22.0; IBM Corp.). Mean values are presented with standard deviations. The clinical, operative, and radiographic variables of the 2 groups were compared using independent-samples t-tests. Significance was set at $\mathrm{p}=$ 0.05 .

\section{Results \\ Demographic and General Clinical Data}

A total of 24 patients with AMC-associated scoliosis and 48 patients with AIS were included in our study (Table 1). The mean patient age at surgery was $14.6 \pm 2.3$ years in the AMC group and $15.0 \pm 2.1$ years in the AIS group $(\mathrm{p}=0.463)$. In the AMC group, there were 7 patients (29.1\%) diagnosed with preoperative comorbidities, including 2 patients with Klippel-Feil syndrome, 1 with facial diplegia, 1 with aortic stenosis, and 3 with pulmonary function impairment. In contrast, in the AIS group, only 1 patient $(2.0 \%)$ who had pulmonary function impairment was diagnosed with a preoperative comorbidity $(29.1 \%$ vs $2.0 \%, \mathrm{p}=0.002)$. There was no significant difference between the 2 groups with regard to Risser grade $(3.2 \pm 2.0$ for AMC patients vs $3.3 \pm 1.7$ for AIS patients) or mean duration of follow-up $(47.8 \pm 10.1$ months for AMC patients versus $49.2 \pm 11.3$ months for AIS patients). The spinal deformities in the AMC group included a single thoracic curve in 16 cases, a single thoracolumbar curve in 5 cases, and thoracic and lumbar curves in 3 cases. In the AIS group, 32 patients had a single thoracic curve, 10 had a single thoracolumbar curve, and 6 had thoracic and lumbar curves. The mean preoperative Cobb angle of the 
TABLE 1. Summary of demographic and clinical characteristics of the 2 groups

\begin{tabular}{lccc}
\hline \multicolumn{1}{c}{ Characteristic } & $\begin{array}{c}\text { AMC Group } \\
(\mathrm{n}=24)\end{array}$ & $\begin{array}{c}\text { AIS Group } \\
(\mathrm{n}=48)\end{array}$ & $\begin{array}{c}\mathrm{p} \\
\text { Value }\end{array}$ \\
\hline Age at surgery (yrs) & & & \\
\hline Mean \pm SD & $14.6 \pm 2.3$ & $15.0 \pm 2.1$ & 0.463 \\
\hline Range & $10-18$ & $10-18$ & \\
\hline Sex (male/female) & $13 / 11$ & $26 / 22$ & \\
\hline Risser grade at surgery & & & \\
\hline Mean \pm SD & $3.2 \pm 2.0$ & $3.3 \pm 1.7$ & 0.825 \\
\hline Range & $1-4$ & $1-4$ & \\
\hline Length of follow-up (mos) & & & 0.610 \\
\hline$\quad$ Mean \pm SD & $47.8 \pm 10.1$ & $49.2 \pm 11.3$ & \\
\hline Range & $24-65$ & $24-71$ & \\
\hline Curve pattern & & & \\
\hline Single thoracic curve & 16 & 32 & \\
\hline Single thoracolumbar curve & 5 & 10 & \\
\hline Thoracic \& lumbar curves & 3 & 6 & \\
\hline
\end{tabular}

coronal major curve was similar in the 2 groups $\left(78.4^{\circ}\right.$ $\pm 17.1^{\circ}$ in the AMC group and $75.1^{\circ} \pm 16.6^{\circ}$ in the AIS group, $\mathrm{p}=0.434$; Table 2 ).

\section{Operative Data}

The mean intraoperative blood loss was $1620 \pm 250 \mathrm{ml}$ in the AMC group and $840 \pm 260 \mathrm{ml}$ in the AIS group ( $\mathrm{p}<0.001)$. The mean duration of the operation was 5.6 \pm 1.6 hours in the AMC group and $4.4 \pm 1.4$ hours in the AIS group $(p=0.002)$. Patients in the AMC group had a mean fusion span of $14.1 \pm 2.3$ levels, versus $12.4 \pm 2.5$ levels in the AIS group $(\mathrm{p}=0.007)$. The mean implant density was $57.3 \% \pm 8.7 \%$ in the AMC group and $68.4 \%$ $\pm 7.9 \%$ in the AIS group ( $<0.001)$. All patients in the AMC group underwent Ponte osteotomy, with the number of levels ranging from 3 to 7 (mean $4.4 \pm 1.2$ levels), while there were only 8 patients in the AIS group who received Ponte osteotomy, with the number of levels ranging from 3 to 4 (mean $3.3 \pm 0.8$ levels).

\section{Radiographic and Clinical Outcomes}

The mean coronal deformity angular ratio was $8.1^{\circ} \pm$ $1.2^{\circ}$ per segment in the AMC group and $10.7^{\circ} \pm 1.9^{\circ}$ per segment in the AIS group $(\mathrm{p}<0.001)$. The mean flexibility of patients in the AMC group tended to be less than that of patients in the AIS group $(23.5 \% \pm 15.1 \%$ vs $48.3 \% \pm$ $16.1 \%$, respectively, $\mathrm{p}<0.001)$. After surgery, the mean initial curve correction was $44.3 \% \pm 11.1 \%$ in the AMC group and $70.8 \% \pm 12.4 \%$ in the AIS group (p < 0.001; Figs. 1 and 2). At the last follow-up, there was a greater mean correction loss in the AMC group than in the AIS group $(5.0 \% \pm 3.1 \%$ vs $2.1 \% \pm 1.9 \%, \mathrm{p}<0.001)$. There were 9 patients in the AMC group with significant pelvic obliquity, all of whom had a single long curve. The mean pelvic obliquity was corrected from $14.2^{\circ} \pm 8.4^{\circ}$ to $4.3^{\circ}$ $\pm 3.2^{\circ}(\mathrm{p}<0.001)$ after surgery and then to $3.8^{\circ} \pm 2.4^{\circ}$ $(\mathrm{p}<0.001)$ at the last follow-up. The mean preoperative
TABLE 2. Comparison of radiographic parameters between the 2 groups

\begin{tabular}{lccr}
\hline Variable & AMC Group & AIS Group & p Value \\
\hline Coronal Cobb angle $\left(^{\circ}\right)$ & & & \\
\hline Preop & $78.4 \pm 17.1$ & $75.1 \pm 16.6$ & 0.434 \\
\hline Postop & $43.7 \pm 9.1$ & $21.9 \pm 7.4$ & $<0.001$ \\
\hline Last follow-up & $47.6 \pm 8.1$ & $23.4 \pm 4.1$ & $<0.001$ \\
\hline Coronal balance $(\mathrm{cm})$ & & & \\
\hline Preop & $1.6 \pm 1.0$ & $1.3 \pm 1.1$ & 0.265 \\
\hline Postop & $1.1 \pm 1.1$ & $0.8 \pm 1.0$ & 0.250 \\
\hline Last follow-up & $0.8 \pm 0.9$ & $0.4 \pm 0.6$ & 0.028 \\
\hline Thoracic kyphosis $\left(^{\circ}\right)$ & & & \\
\hline Preop & $-19.2 \pm 13.3$ & $15.2 \pm 7.1$ & $<0.001$ \\
\hline Postop & $11.2 \pm 8.4$ & $23.2 \pm 6.4$ & $<0.001$ \\
\hline Last follow-up & $8.7 \pm 7.4$ & $21.6 \pm 7.1$ & $<0.001$ \\
\hline Lumbar lordosis $\left(^{\circ}\right)$ & & & \\
\hline Preop & $-54.4 \pm 8.1$ & $-46.3 \pm 5.3$ & $<0.001$ \\
\hline Postop & $-43.6 \pm 5.8$ & $-42.1 \pm 6.1$ & 0.321 \\
\hline Last follow-up & $-46.9 \pm 6.4$ & $-44.9 \pm 4.9$ & 0.146 \\
\hline SVA (cm) & & & \\
\hline Preop & $5.6 \pm 2.1$ & $2.1 \pm 1.8$ & $<0.001$ \\
\hline Postop & $2.1 \pm 1.7$ & $1.2 \pm 1.0$ & 0.006 \\
\hline Last follow-up & $2.3 \pm 1.2$ & $1.7 \pm 1.1$ & 0.038 \\
\hline Vas are prent & & & \\
\hline
\end{tabular}

Values are presented as the mean $\pm S D$ unless stated otherwise.

thoracic kyphosis was $-19.2^{\circ} \pm 13.3^{\circ}$ in the AMC group and $15.2^{\circ} \pm 7.1^{\circ}$ in the AIS group $(\mathrm{p}<0.001)$, then it corrected to $11.2^{\circ} \pm 8.4^{\circ}$ in the AMC group and $23.2^{\circ} \pm 6.4^{\circ}$ in the AIS group immediately after surgery $(\mathrm{p}<0.001)$. The mean thoracic kyphosis at the most recent follow-up visit was $8.7^{\circ} \pm 7.4^{\circ}$ in the $\mathrm{AMC}$ group and $21.6^{\circ} \pm 7.1^{\circ}$ in the AIS group $(\mathrm{p}<0.001)$.

At the last follow-up, the AMC group had lower SRS22 scores than the AIS group in the domains of pain (4.0 vs $4.3, p=0.004$ ), self-image ( 3.9 vs $4.5, p<0.001$ ), function (4.0 vs $4.8, \mathrm{p}<0.001)$, and mental health (4.1 vs $4.5, \mathrm{p}$ $=0.001$ ); however, the AMC group showed improvement in each domain (Table 3).

\section{Analysis of Pedicle Screw Placement}

In the AMC group, 57 (15.9\%) of 357 screws were found to have perforated at least 1 pedicle wall or vertebral cortical wall, as compared with $76(9.5 \%)$ of 796 screws in the AIS group ( $p=0.002$; Table 4). Of the 57 perforations in the AMC group, 25 (43.9\%) were lateral perforations, $13(22.8 \%)$ were medial, and $19(33.3 \%)$ were anterior; $38(66.7 \%)$ of the perforations were grade 1 perforation, $16(28.1 \%)$ were grade $2,2(3.5 \%)$ were grade 3 , and $1(1.8 \%)$ was grade 4 . In $51(89.5 \%)$ of the instances of perforation in the AMC group, the screws were regarded as acceptably positioned and in the remaining $6(10.5 \%)$ the screw position was considered unacceptable. None of these unacceptably malpositioned screws needed revision, however, due to absence of impingement on the aorta, trachea, or other visceral organs. 

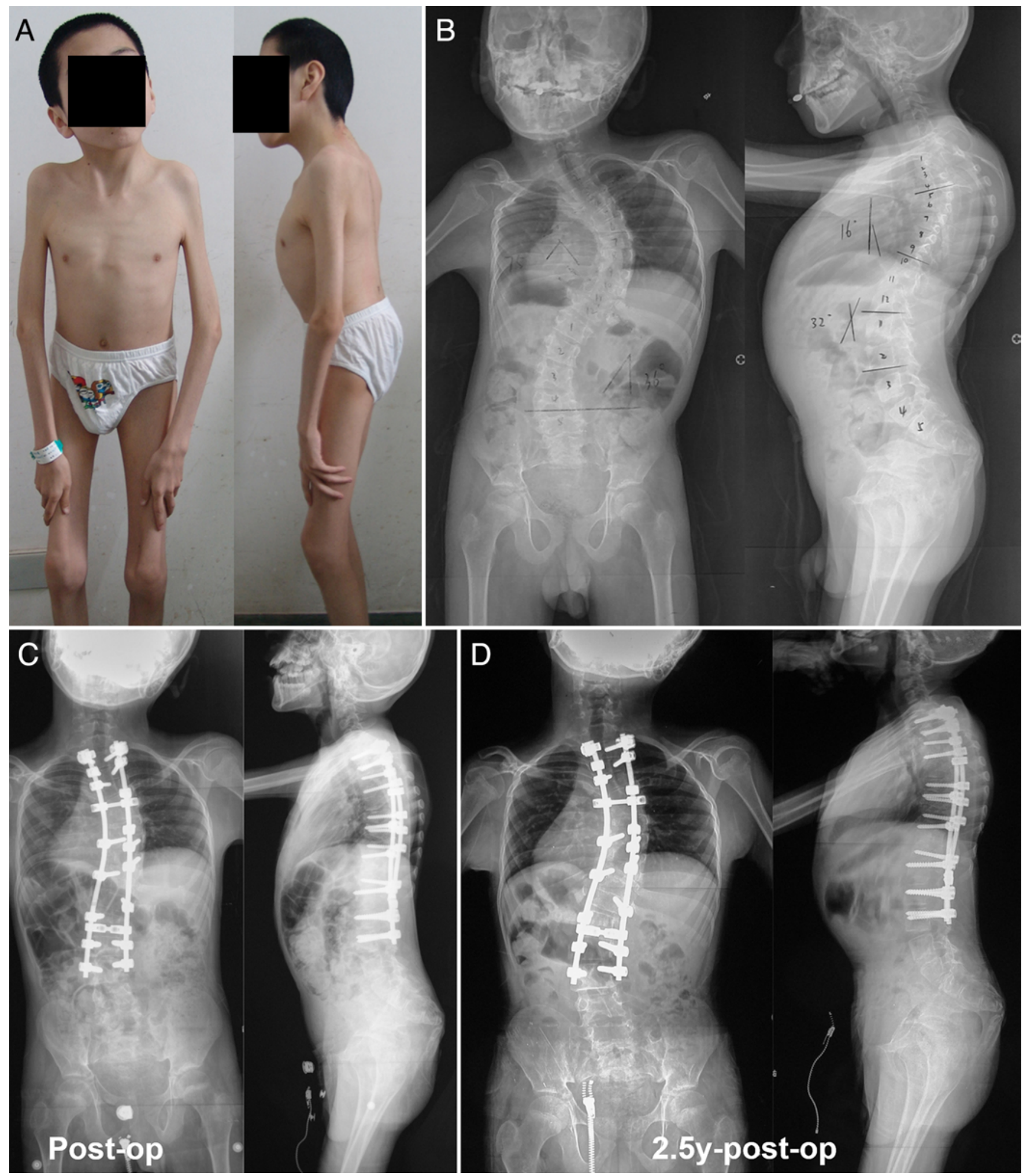

FIG. 1. Representative case of AMC-associated scoliosis. This boy presented at the age of 14 years with AMC complicated with scoliosis. As shown in the photographs obtained before surgery (A), he had elbow and knee contractures. Preoperative anteroposterior and lateral radiographs (B) showed severe scoliosis in the coronal plane as well as a hyperlordotic lumbar spine in the sagittal plane. Ponte osteotomies were performed from T6 to T11 and fusion from T2 to L3 (C). Postoperatively, the coronal Cobb angle of the major curve decreased from $75^{\circ}$ to $43^{\circ}$, resulting in significant improvement of his sagittal alignment. Follow-up radiographs obtained 2.5 years after surgery (D) showed that the sagittal alignment was well maintained, although some loss of scoliosis correction was noted in the coronal plane. Figure is available in color online only. 

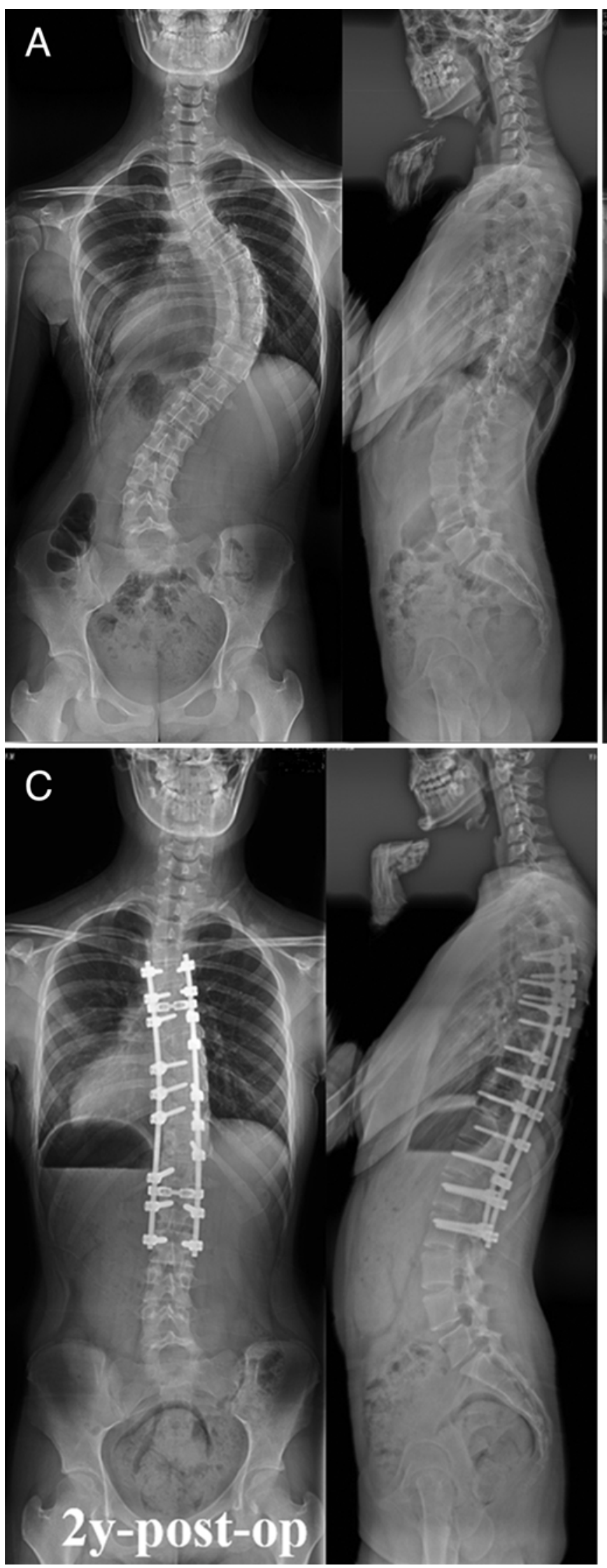

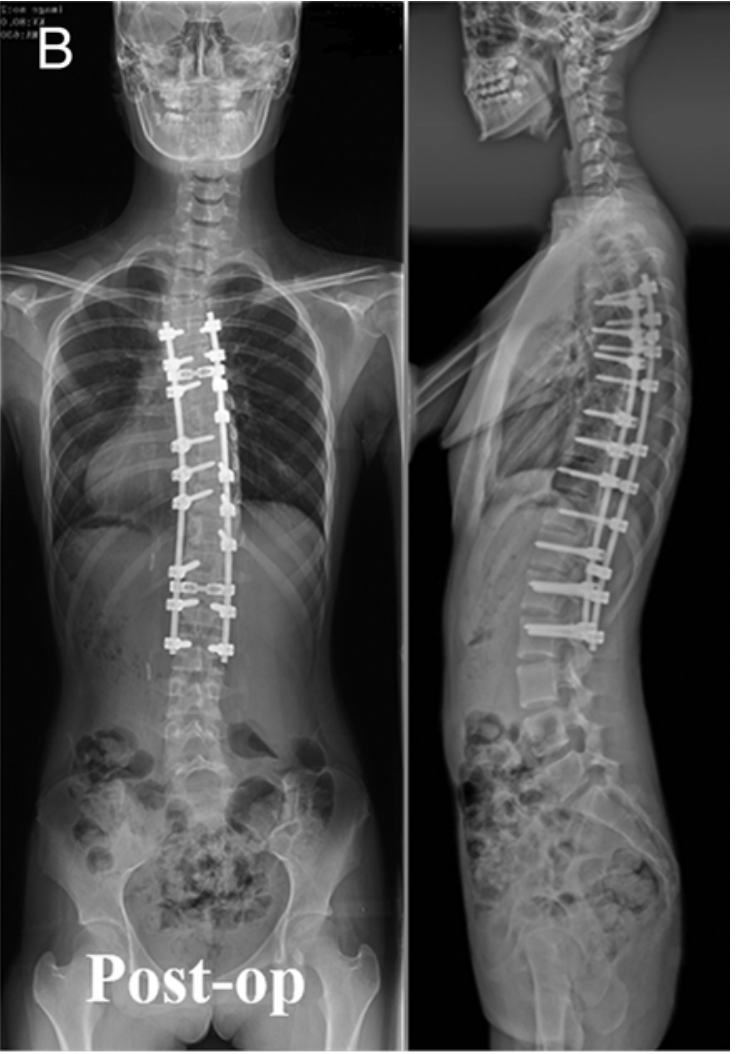

FIG. 2. Representative case of AIS. This girl presented at the age of 13 years with AIS (preoperative radiographs, A). She was treated with posterior instrumentation and fusion from T4 to L2 (B). Comparison of preoperative and postoperative radiographs showed that the coronal Cobb angle of the major curve decreased from $77^{\circ}$ to $22^{\circ}$. Follow-up radiographs obtained 2 years after surgery $(\mathbf{C})$ showed good maintenance of both coronal and sagittal alignment. 
TABLE 3. Comparison of SRS-22 scores between the 2 groups

\begin{tabular}{cccc}
\hline Variable & AMC Group & AIS Group & p Value \\
\hline Pain & & & \\
\hline Preop & $3.8 \pm 0.5$ & $4.2 \pm 0.6$ & 0.006 \\
\hline Last follow-up & $4.0 \pm 0.4$ & $4.3 \pm 0.4$ & 0.004 \\
\hline Self-image & & & \\
\hline Preop & $3.3 \pm 0.3$ & $4.1 \pm 0.4$ & $<0.001$ \\
\hline Last follow-up & $3.9 \pm 0.5$ & $4.5 \pm 0.5$ & $<0.001$ \\
\hline Function & & & \\
\hline Preop & $3.6 \pm 0.5$ & $4.3 \pm 0.6$ & $<0.001$ \\
\hline Last follow-up & $4.0 \pm 0.6$ & $4.8 \pm 0.3$ & $<0.001$ \\
\hline Mental & & & \\
\hline Preop & $3.9 \pm 0.3$ & $4.2 \pm 0.4$ & 0.002 \\
\hline Last follow-up & $4.1 \pm 0.4$ & $4.5 \pm 0.5$ & 0.001 \\
\hline Satisfaction & & & \\
\hline Preop & NA & NA & \\
\hline Last follow-up & $4.2 \pm 0.3$ & $4.4 \pm 0.3$ & 0.010 \\
\hline
\end{tabular}

$\mathrm{NA}=$ not applicable

Values are presented as the mean \pm SD unless stated otherwise.

\section{Complications}

The complication rate was much higher in the AMC group than in the AIS group (33.3\% [8/24] vs 8.3\% [4/48]), $\mathrm{p}=0.016]$. Eight complication events were noted in the AMC group. One AMC patient experienced somatosensory evoked potential and motor evoked potential signals loss in bilateral lower extremities after osteotomy. The wake-up test revealed that the patient's lower limb muscle strength had dropped to grade $2 / 5$. The rods were loosened and retightened without forcible correction after removal of the gelatin sponges that had been applied for hemostasis at the osteotomy site. At 2 months' follow-up, the weakness in the lower limbs had fully resolved. In 1 AMC case, the patient had temporary perioperative dyspnea, which was managed by delaying extubation. One AMC patient who experienced malignant hyperthermia was resuscitated through an intensive rescue effort. Three AMC patients had pleural effusions due to screw perforations. The distal adding-on phenomenon was noted in 1 patient in the AMC group and in 3 patients in the AIS group. There was 1 patient with proximal junctional kyphosis in each group. No instrumentation complications, such as broken screws, broken rods, or screw dislodgement, were found in either group. No infection or pseudarthrosis was noted. No additional revision surgery was required in either group as of the most recent follow-up.

\section{Discussion}

As scoliosis in AMC is unusual and the number of cases reviewed in previous studies is also relatively small, ${ }^{16}$ there is no study that has directly compared the results of spinal deformity correction between AMC and AIS patients. This study showed that spinal deformity correction in patients with AMC differed in several ways from that in patients with AIS.

Our findings revealed a significantly longer operation
TABLE 4. Comparison of screw malpositioning between the 2 groups

\begin{tabular}{lccc}
\hline \multicolumn{1}{c}{ Variable } & AMC Group & AIS Group & p Value \\
\hline $\begin{array}{l}\text { Total no. of implants (screws \& } \\
\text { hooks) }\end{array}$ & 388 & 814 & \\
\hline No. of pedicle screws & 357 & 796 & \\
\hline No. of malpositioned screws & 57 & 76 & \\
\hline Rate of screw malpositioning & $16.0 \%$ & $9.5 \%$ & 0.003 \\
\hline Type of perforation & & & 0.823 \\
\hline Lateral & $25(43.9 \%)$ & $35(46.1 \%)$ & \\
\hline Medial & $13(22.8 \%)$ & $14(18.4 \%)$ & \\
\hline Anterior & $19(33.3 \%)$ & $27(35.5 \%)$ & \\
\hline Grade of perforation & & & 0.108 \\
\hline Grade 1 & $38(66.7 \%)$ & $62(81.6 \%)$ & \\
\hline Grade 2 & $16(28.1 \%)$ & $13(17.1 \%)$ & \\
\hline Grade 3 & $2(3.5 \%)$ & $1(1.3 \%)$ & \\
\hline Grade 4 & $1(1.8 \%)$ & 0 & \\
\hline Acceptability of screw position* & & & 0.042 \\
\hline Acceptable & $51(89.5 \%)$ & $75(98.7 \%)$ & \\
\hline Unacceptable & $6(10.5 \%)$ & $1(1.3 \%)$ & \\
\hline * Number of screws (\%). & & & \\
\hline
\end{tabular}

time and more intraoperative blood loss in the AMC group compared with the AIS group. The reason is that the spinal deformity in AMC patients is rigid, as demonstrated by the side-bending radiographs (mean flexibility $23.8 \% \pm$ $14.7 \%$ ). To achieve better correction, posterior osteotomy was performed but this increased the intraoperative blood loss. Importantly, the AMC patients had a hyperlordotic sagittal alignment in addition to taut soft tissues, both of which pose substantial challenges to exposure of the spine and lead to increased damage to paraspinal muscles and increased bleeding. Most strikingly, surgical correction in the AMC group involved more instrumented levels. We believe that the fusion of more levels in AMC patients than in AIS patients may be attributed to 3 factors. First, most of the patients with AMC-associated scoliosis had a long curve. Second, as mentioned above, an abnormal sagittal profile is common in AMC-associated scoliosis, and this can only be addressed by long fusion. Third, the spinal deformity in AMC is related to difficulty in screw placement. In our study, we found a higher rate of screw malpositioning in the AMC group than in the AIS group $(16.0 \%$ vs $9.5 \%, p=0.003)$. Due to the challenges of pedicle screw placement in AMC patients, extending the fusion is helpful to increase the spinal anchors and to enhance the instrumentation stability. For the higher intraoperative blood loss in the AMC group, pre-deposited autotransfusion, intraoperative autologous blood transfusion, and application of tranexamic acid should be considered among the options for special perioperative care.

The mean correction rate in the AMC group was $44.3 \% \pm 11.1 \%$, in contrast to $70.8 \% \pm 12.4 \%$ in the AIS group. In their study of scoliosis in AMC, Yingsakmongkol and Kumar reported a correction rate of $36.5 \%$ for patients treated with posterior spinal arthrodesis with instrumentation. ${ }^{22}$ We were able to achieve a slightly higher 
rate with posterior multisegmental release in our AMC patients, but we still could not reach the level achieved in the AIS patients. ${ }^{14}$ In addition to thinner pedicles and greater difficulty in screw placement, the AMC group had a significantly lower mean implant density than the AIS group $(57.3 \% \pm 8.7 \%$ vs $68.4 \% \pm 7.9 \%, \mathrm{p}<0.001)$ in our study. For AIS patients, Yang et al. ${ }^{21}$ found that implant density was not related to radiographic and cosmetic outcomes. However, for the treatment of rigid curves in AMC patients, higher implant density means greater correction strength, not only achieving better correction outcome through the correction surgery but also meaningful for the maintenance of correction outcome during the followup period. As revealed by our findings, although no instrumentation failure had occurred as of the most recent follow-up, the mean loss of correction was greater in the AMC group than in the AIS group $(5.0 \% \pm 3.1 \%$ vs $2.1 \%$ $\pm 1.9 \%, \mathrm{p}<0.001$ ).

Other coronal plane differences between AMC and AIS curves have been previously identified. Herron et al. ${ }^{13}$ reported that 16 patients (89\%) in their cohort had pelvic obliquity and noted that when pelvic obliquity developed, it was a serious sign and the progression of pelvic obliquity was coincident with curve progression. Pelvic obliquity in arthrogryposis seems to be the result of contracture of soft tissue as well as muscle imbalance above and below the pelvis. Herron et al. recommended fusion of the spine to the sacrum for those patients whose scoliosis and pelvic obliquity were progressive despite attempts at surgical correction of the associated deformities of the extremities. In reviewing the literature, we found that in the cohort reported on by Yingsakmongkol and Kumar, 13 (81\%) of 16 AMC cases were associated with pelvic obliquity; in 12 of these 13 cases, the patients presented with a single thoracolumbar curve and only 1 patient presented with thoracolumbar double curves. ${ }^{22}$ In our study, 9 (38\%) of 24 AMC patients also had pelvic obliquity, and all 9 had a single long curve. Therefore, we propose that pelvic obliquity is also closely related to the long C-type curve in AMC. Asymmetrical contracture of the paraspinal muscles, especially those below the pelvis, may result in lifting of the pelvis on the concave side. And we believe the correction surgery may release the paraspinal muscles of the concave side and balance the pelvis. In this study, we only performed spinal correction surgery and balanced the pelvis to a certain extent, without extending fusion to the pelvis.

With regard to sagittal alignment, there was an apparent difference between the AMC and AIS patients. Thoracic AIS is characterized by thoracic hypokyphosis or even lordosis, whereas deformity in AMC is characterized by thoracolumbar hyperlordosis associated with a forward leaning of the trunk, which may be due to hip flexion deformities. ${ }^{13}$ Consequently, thoracic hyperlordosis could shorten the anteroposterior diameter of the thorax, affecting cardiopulmonary system development. ${ }^{3}$ Moreover, under such conditions, patients could easily have back pain due to muscle fatigue. ${ }^{8}$ Therefore, correction of sagittal plane alignments in AMC should be considered in the surgical decision-making process. In our study, thoracic kyphosis in AMC patients was restored by lifting the spine backward, and trunk balance was also improved.
Moreover, we acknowledge that the fusion of more levels in patients with AMC would contribute to a better overall sagittal correction.

The incidence of pedicle screw misplacement in the AMC group was higher than that in the AIS group. Thus, intraoperative spinal navigation may be considered for the purpose of improving accuracy of screw placement in AMC patients. Moreover, hooks could be used instead if the pedicles are too thin for screws. In addition to 4 cases of pedicle screw malpositioning, there was also 1 case in the AMC group in which there was a loss of SEP and MEP signals during osteotomy and the patient demonstrated decreased lower limb muscle strength on the intraoperative wake-up test. In treating patients with AMC, it is not just the risks associated with surgical technique but also concerns related to perioperative care that should be taken into consideration. Of primary concern are the potential for difficulties with tracheal intubation, cervical spine abnormalities, chronic lung disease related to recurrent bouts of aspiration or restriction due to scoliosis, difficulties with venous access, and malignant hyperthermia. ${ }^{18}$ In our cohort of AMC patients, the prevalence of preoperative comorbidities was significantly higher than in the AIS group. Moreover, one patient had temporary dyspnea and another had malignant hyperthermia during the perioperative period.

Several limitations of this study should be addressed. First, patients with vertebral anomalies were excluded from this study with the purpose of better matching the radiographs and surgical procedures between the 2 groups. Second, comparison of fusion rate as well as postoperative pulmonary function between the 2 groups was limited due to the retrospective nature of this study and the fact that although all patients underwent preoperative and immediate postoperative CT, scans were performed during follow-up only when there was some suspicion of implant dislodgement and/or notching. However, our study had several strengths when compared with the existing literature. First, we had a relatively large cohort of patients with AMC, and we directly compared outcomes for those patients with outcomes for patients with AIS during a contemporaneous period. Second, this study matched patients with AIS and patients with AMC at a 2:1 ratio to improve the study's statistical strength. Third, all patients in this single-center study were treated by surgeons working with the same surgical decision making, which minimized the variations in operative strategies and techniques between patients and thus maximally guaranteed the consistency of surgical results.

\section{Conclusions}

Correction of scoliosis in patients with AMC tends to require more operating time and fusion of more vertebral levels than AIS and results in less correction, more blood loss, and more complications. In addition, more attention should be paid to pelvic obliquity and sagittal hyperlordosis in AMC patients. We hope that this information can help improve the safety and efficacy of surgical management of scoliosis in patients with AMC.

\section{Acknowledgments}

This work was supported by the National Natural Science Foun- 
dation of China (grant no. 81772422) and the Natural Science Foundation of Jiangsu Province (grant no. BE2017606).

\section{References}

1. Amiot LP, Lang K, Putzier M, Zippel H, Labelle H: Comparative results between conventional and computer-assisted pedicle screw installation in the thoracic, lumbar, and sacral spine. Spine (Phila Pa 1976) 25:606-614, 2000

2. Astur N, Flynn JM, Flynn JM, Ramirez N, Glotzbecker M, van Bosse HJ, et al: The efficacy of rib-based distraction with VEPTR in the treatment of early-onset scoliosis in patients with arthrogryposis. J Pediatr Orthop 34:8-13, 2014

3. Bevan WP, Hall JG, Bamshad M, Staheli LT, Jaffe KM, Song $\mathrm{K}$ : Arthrogryposis multiplex congenita (amyoplasia): an orthopaedic perspective. J Pediatr Orthop 27:594-600, 2007

4. Brown LM, Robson MJ, Sharrard WJ: The pathophysiology of arthrogryposis multiplex congenita neurologica. J Bone Joint Surg Br 62:291-296, 1980

5. Daher YH, Lonstein JE, Winter RB, Moe JH: Spinal deformities in patients with arthrogryposis. A review of 16 patients. Spine (Phila Pa 1976) 10:609-613, 1985

6. Drummond DS, Mackenzie DA: Scoliosis in arthrogryposis multiplex congenita. Spine (Phila Pa 1976) 3:146-151, 1978

7. Fassier A, Wicart P, Dubousset J, Seringe R: Arthrogryposis multiplex congenita. Long-term follow-up from birth until skeletal maturity. J Child Orthop 3:383-390, 2009

8. Galbusera F, Wilke HJ, Brayda-Bruno M, Costa F, Fornari $\mathrm{M}$ : Influence of sagittal balance on spinal lumbar loads: a numerical approach. Clin Biomech (Bristol, Avon) 28:370377, 2013

9. Gill JB, Levin A, Burd T, Longley M: Corrective osteotomies in spine surgery. J Bone Joint Surg Am 90:2509-2520, 2008

10. Greggi T, Lolli F, Di Silvestre M, Martikos K, Vommaro F, Maredi E, et al: Complications incidence in the treatment of early onset scoliosis with growing spinal implants. Stud Health Technol Inform 176:334-337, 2012

11. Greggi T, Martikos K, Pipitone E, Lolli F, Vommaro F, Maredi E, et al: Surgical treatment of scoliosis in a rare disease: arthrogryposis. Scoliosis 5:24, 2010

12. Hall JG: Arthrogryposis multiplex congenita: etiology, genetics, classification, diagnostic approach, and general aspects. J Pediatr Orthop B 6:159-166, 1997

13. Herron LD, Westin GW, Dawson EG: Scoliosis in arthrogryposis multiplex congenita. J Bone Joint Surg Am 60:293299, 1978

14. Holewijn RM, Schlösser TP, Bisschop A, van der Veen AJ, Stadhouder A, van Royen BJ, et al: How does spinal release and ponte osteotomy improve spinal flexibility? The law of diminishing returns. Spine Deform 3:489-495, 2015
15. Joo S, Rogers KJ, Donohoe M, King MM, Kumar SJ: Prevalence and patterns of scoliosis in children with multiple pterygium syndrome. J Pediatr Orthop 32:190-195, 2012

16. Komolkin I, Ulrich EV, Agranovich OE, van Bosse HJP: Treatment of scoliosis associated with arthrogryposis multiplex congenita. J Pediatr Orthop 37 (Suppl 1):S24-S26, 2017

17. Lehman RA Jr, Lenke LG, Keeler KA, Kim YJ, Cheh G: Computed tomography evaluation of pedicle screws placed in the pediatric deformed spine over an 8-year period. Spine (Phila Pa 1976) 32:2679-2684, 2007

18. Martin S, Tobias JD: Perioperative care of the child with arthrogryposis. Paediatr Anaesth 16:31-37, 2006

19. Siebold RM, Winter RB, Moe JH: The treatment of scoliosis in arthrogryposis multiplex congenita. Clin Orthop Relat Res (103):191-198, 1974

20. Smith-Petersen MN, Larson CB, Aufranc OE: Osteotomy of the spine for correction of flexion deformity in rheumatoid arthritis. Clin Orthop Relat Res 66:6-9, 1969

21. Yang S, Jones-Quaidoo SM, Eager M, Griffin JW, Reddi V, Novicoff W, et al: Right adolescent idiopathic thoracic curve (Lenke $1 \mathrm{~A}$ and B): does cost of instrumentation and implant density improve radiographic and cosmetic parameters? Eur Spine J 20:1039-1047, 2011

22. Yingsakmongkol W, Kumar SJ: Scoliosis in arthrogryposis multiplex congenita: results after nonsurgical and surgical treatment. J Pediatr Orthop 20:656-661, 2000

23. Zhu F, Sun X, Qiao J, Ding Y, Zhang B, Qiu Y: Misplacement pattern of pedicle screws in pediatric patients with spinal deformity: a computed tomography study. J Spinal Disord Tech 27:431-435, 2014

\section{Disclosures}

The authors report no conflict of interest concerning the materials or methods used in this study or the findings specified in this paper.

\section{Author Contributions}

Conception and design: Xu, Z Chen. Acquisition of data: Z Chen, X Chen, Du. Analysis and interpretation of data: Xu, Z Chen, Li, Zhou. Drafting the article: Xu. Critically revising the article: Sun, Qiu. Statistical analysis: Xu. Study supervision: Sun, Qiu.

\section{Correspondence}

Xu Sun: Drum Tower Hospital, Nanjing University Medical School, Nanjing, China. drsunxu@163.com. 